\title{
COMPACT LINEAR TRANSFORMATIONS
}

\author{
C. T. TAAM
}

1. Consider a compact linear transformation $T$ (also called completely continuous transformation) from a Banach space $A$ to a Banach space $B$. Can $T$ be approximated arbitrarily close in norm by bounded linear transformations whose ranges are finite dimensional (see $[1$, p. 49])? The answer is affirmative for the following types of domain and range spaces: (i) both $A$ and $B$ are Hilbert spaces (see [2, p. 204]), (ii) both $A$ and $B$ are $C[a, b]$ (see [2, p. 222] or [3]), (iii) there is no other restriction on $A$, but $B$ is of "type $A$ " [4], (iv) $A$ is either $L^{p}$ or $C$ and there is no other restriction on $B$ (see [5, p. 536]). In this paper we shall show that the answer is also affirmative when $A$ is any Banach space and $B$ is $C(E), E$ being a compact Hausdorff space.

Let $S^{*}$ be the strongly closed unit sphere in the conjugate space $B^{*}$, namely the set of all linear functionals of unit norm or less. $S^{*}$ is a compact Hausdorff space in the relative topology introduced in $S^{*}$ by the weak * topology of $B^{*}$ (see [1, p. 37]). For convenience, we continue to call this relative topology in $S^{*}$ the weak $*$ topology. Denote by $C\left(S^{*}\right)$ the Banach algebra of all the complex-valued weak *-continuous functions in $S^{*}$. For each $x$ in $B$, the mapping $x \rightarrow x^{* *}$ is an isometric isomorphism embedding $B$ as a subspace of $B^{* *}$, and $x^{* *} \rightarrow x^{* *}$ (restricted to $S^{*}$ ) is also an isomorphism satisfying

$$
\left\|x^{* *}\right\|=\underset{F \in S^{*}}{\text { l.u.b. }}\left|x^{* *}(F)\right|=\left\|x^{* *}\right\|_{\infty}
$$

where $\left\|x^{* *}\right\|_{\infty}$ is the uniform norm of $x^{* *}$ restricted to $S^{*}$. Hence we can embed $B$ as a subspace of $C\left(S^{*}\right)$ under the isometric isomorphism $x \rightarrow x^{* *}$ (restricted to $S^{*}$ ). Consequently, by embedding $B$ in $C\left(S^{*}\right)$, a compact linear transformation $T$ from a Banach space $A$ to a Banach space $B$ can be approximated arbitrarily close in norm by bounded linear transformations of finite dimensional range from $A$ to $C\left(S^{*}\right)$. (See $\S 3$.)

The ideas in $\$ \S 2$ and 3 are suggested by those of Radon in [3]; (see also [2, p. 222]). Throughout this note, $C(E)$ denotes the Banach algebra of all complex-valued continuous functions defined on a compact Hausdorff space $E$, and $\left(T^{*} F\right)(x)$ means $(T x)(F)$, for $x$ in $A$, $T x$ in $C(E)$ and $F$ in $E$.

Presented to the Society, January 22,1959, under the title On compact linear transformations in Banach space; received by the editors April 8, 1959. 
2. In this section we shall establish the following result:

Let $T$ be a compact linear transformation from a Banach space $A$ to the space $C(E)$. Then $T^{*}$ is a continuous mapping on $E$ to $A^{*}$. (Here $A^{*}$ is given the usual norm topology.)

Proof. Take a fixed $F_{0}$ in $E$. To each $\epsilon>0$, we have to show that there is an open set $O$ in $E$ containing $F_{0}$ such that

$$
\left\|T^{*} F-T^{*} F_{0}\right\|<\epsilon \quad \text { for all } F \in O .
$$

Suppose that this is not true. Then there is an $\epsilon=2 \epsilon_{0}>0$ such that (1) is satisfied by no open set $O$ in $E$ containing $F_{0}$. We show that it leads to contradiction.

By virtue of the compactness of $T$, the image $T(S)$ of the closed unit sphere $S$ in $A$ is separable and hence contains a sequence $\left\{z_{n}\right\}$ dense in the closure of $T(S)$.

The sets $U_{m, n}=\left\{F|| z_{m}(F)-z_{m}\left(F_{0}\right) \mid<1 / n, F \in E\right\}$ are open sets in $E$ and form a sequence $\left\{V_{k}\right\}$. Let $O_{n}$ be the intersection of $V_{1}$, $V_{2}, \cdots$ and $V_{n}$. Clearly $F_{0}$ lies in each $O_{n}$ and $\left\{O_{n}\right\}$ is monotonic decreasing. By the supposition, for each $n$, there exists a $F_{n}$ in $O_{n}$ such that $\left\|T^{*} F_{n}-T^{*} F_{0}\right\| \geqq 2 \epsilon_{0}$. But then there is a $x_{n}$ in $S$ such that

$$
\left|\left[T x_{n}\right]\left(F_{n}\right)-\left[T x_{n}\right]\left(F_{0}\right)\right|=\left|\left[T^{*} F_{n}-T^{*} F_{0}\right]\left(x_{n}\right)\right|>\epsilon_{0} .
$$

Since $T$ is compact, we may suppose, by passing to a subsequence if necessary, that

$$
T x_{n}=y_{n} \text { converges in norm to some } y \text { in } C(E) .
$$

As $y$ can be approximated arbitrarily close in norm by $\left\{z_{n}\right\}$, to an integer $p$ satisfying $3<\epsilon_{0} p$, there is an integer $q$ such that

$$
\left\|y-z_{q}\right\|_{\infty}<\frac{1}{p}<\frac{\epsilon_{0}}{3} .
$$

Let $N$ be so large that $O_{N}$ is contained in $U_{q, p}$. Then by (4), $\left|y\left(F_{n}\right)-z_{q}\left(F_{n}\right)\right|<1 / p,\left|y\left(F_{0}\right)-z_{q}\left(F_{0}\right)\right|<1 / p$ and, for $n \geqq N$, $\left|z_{q}\left(F_{n}\right)-z_{q}\left(F_{0}\right)\right|<1 / p$. Hence

$$
\left|y\left(F_{n}\right)-y\left(F_{0}\right)\right|<3 / p<\epsilon_{0} \quad \text { for } n \geqq N .
$$

Now that, by virtue of (3), $\left|y_{n}\left(F_{n}\right)-y\left(F_{n}\right)\right|$ and $\left|y_{n}\left(F_{0}\right)-y\left(F_{0}\right)\right|$ both tend to zero as $n \rightarrow \infty$ and (5) together show that (2) cannot hold for all $n$. The contradiction proves that $T^{*}$ is continuous on $E$.

3. Let $T$ be the transformations of $\$ 2$. For each $F_{k}$ in $E$ and each $\epsilon>0$, the set $O_{k}=\left\{F \mid\left\|T^{*} F-T^{*} F_{k}\right\|<\epsilon, F \in E\right\}$ is open. Let $g_{k}$ be a real-valued continuous function in $E$ such that $g_{k}=2$ at $F_{k}, g_{k}=0$ 
outside $O_{k}, 0 \leqq g_{k} \leqq 2$. The existence of such functions is assured by the Urysohn's lemma. Set $U_{k}=\left\{F \mid g_{k}(F)>1, F\right.$ in $\left.E\right\}$, then $U_{k}$ is an open set containing $F_{k}$, and $U_{k} \subset O_{k}$. Since $E$ is compact, it can be covered by some finite family of sets $U_{1}, U_{2}, \cdots, U_{n}$. Setting $h_{1}(F)$ $=\inf \left(g_{1}(F), 1\right)$, we define inductively

$$
h_{m}=\inf \left(\sum_{i=1}^{m-1} h_{i}+g_{m}, 1\right)-\sum_{i=1}^{m-1} h_{i}, \quad m=2,3, \cdots, n .
$$

The functions $h_{m}$ are continuous and belong to $C(E)$. They satisfy

$$
o \leqq h_{i}(F) \leqq 1, \quad \sum_{i=1}^{n} h_{i}(F)=1 \text { in } E,
$$

$h_{i}(F) \neq 0$ implies $F \in O_{i}$. For $x$ in $A$, define

$$
T_{n} x=\sum_{i=1}^{n}(T x)\left(F_{i}\right) h_{i}
$$

Clearly $T_{n} x$ is in $C(E)$ and the range of $T_{n}$ is finite dimensional. Using the properties of the functions $h_{i}$ and the definition of $O_{i}$, we can see that in $E$

$$
\begin{aligned}
\left|(T x)(F)-\left(T_{n} x\right)(F)\right| & =\left|\left(T^{*} F\right)(x)-\sum_{i=1}^{n} h_{i}(F)\left(T^{*} F_{i}\right)(x)\right| \\
& \leqq\left\|T^{*} F-\sum_{i=1}^{n} h_{i}(F) T^{*} F_{i}\right\|\|x\| \\
& \leqq \sum_{i=1}^{n} h_{i}(F)\left\|T^{*} F-T^{*} F_{i}\right\|\|x\| \\
& <\epsilon\|x\| .
\end{aligned}
$$

Hence

$$
\left\|T x-T_{n} x\right\|_{\infty}<\epsilon\|x\|, \quad\left\|T-T_{n}\right\| \leqq \epsilon .
$$

We have thus proved the following result:

A compact linear transformation $T$ from a Banach space $A$ to the space $C(E)$ can be approximated arbitrarily close in norm by bounded linear transformations of finite-dimensional range.

In view of the discussion in $\$ 1$, it follows that

A compact linear transformation $T$ from a Banach space $A$ to a Banach space $B$, embedded in $C\left(S^{*}\right)$, can be approximated arbitrarily close in norm by bounded linear transformations of finite-dimensional range from $A$ to $C\left(S^{*}\right)$. 
4. If a sequence of compact linear transformations converges to a limit in norm it is known that the limit is compact. (See [1, p. 49]). In view of this property, the first result in $\$ 3$ can be stated as follows:

$T$ is a compact linear transformation from a Banach space $A$ to a Banach algebra $C(E)$ if and only if $T$ can be approximated arbitrarily close in norm by bounded linear transformations of finite-dimensional range from $A$ to $C(E)$.

The method in $\S 3$ uses essentially the continuity of $T^{*}$. Hence from $\S \S 2$ and 3 we see that

A bounded linear transformation $T$ from a Banach space $A$ to a Banach algebra $C(E)$ is compact if and only if $T^{*}$ is continuous from $E$ to $A^{*}$.

As consequences of these remarks we also see that

A linear transformation from a Banach space $A$ to a Banach space $B$ is compact if and only if when $B$ is embedded in $C\left(S^{*}\right)$ it can be approximated arbitrarily close in norm by bounded linear transformations of finite-dimensional range from $A$ to $C\left(S^{*}\right)$; and

A bounded linear transformation $T$ from a Banach space $A$ to a Banach space $B$ is compact if and only if $T^{*}$ is weak ${ }^{*}$-continuous on $S^{*}$ to $A^{*}$.

Let $\beta(A, B)\left(\beta\left(A, C\left(S^{*}\right)\right)\right)$ be the Banach space of all compact linear transformations from the Banach space $A$ to the Banach space $B\left(C\left(S^{*}\right)\right)$. We can also express the above results in the following form:

$\beta(A, B)$ can be embedded in $\beta\left(A, C\left(S^{*}\right)\right)$. The subspace of all the transformations of finite-dimensional range in $\beta\left(A, C\left(S^{*}\right)\right)$ is dense in $\beta\left(A, C\left(S^{*}\right)\right)$.

When $A=B, \beta(A, A)$ is an algebra. To apply the results above, we can embed both the domain $A$ and range $A$ in $C\left(S^{*}\right)$.

We observe that the completeness of $A$ has not been used in this note.

\section{REFERENCES}

1. E. Hille and R. S. Phillips, Functional analysis and semi-groups, Amer. Math. Soc. Colloquium Publications, vol. 31, 1957.

2. R. Riesz and B. Sz-Nagy, Functional analysis, New York, 1955.

3. J. Radon, Über Lineare Funktionaltransformationen und Funktionalgleichungen, Sitzsber. Akad. Wiss. Wien. vol. 128 (1919) pp. 1083-1121.

4. I. Maddaus, On completely continuous linear transformations, Bull. Amer. Math. Soc. vol. 44 (1938) pp. 279-282.

5. R. S. Phillips, On linear transformations, Trans. Amer. Math. Soc. vol. 48 (1940) pp. 516-541.

\section{GeORGETOWN UnIVERSITy}

